\section{Who does what, and how much in the preschool child health services in England}

\section{J A MACFARLANE, UMSIE PILLAY}

\section{Introduction}

The Royal College of General Practitioners has recently proposed that child health surveillance in England should be carried out by general practitioners.' It has become apparent, however, from personal communications from district medical officers in certain areas that there is already considerable variation in who does what, and how much in the child health services in different districts. For example, from initial inquiries it was apparent that $73^{\circ}$; of child health clinics in the Oxford district were carried out by general practitioners, $91^{\circ}{ }^{\circ}$ of child health clinics in East Suffolk were carried out by health visitors, whereas in Croydon $100^{\circ}{ }^{\prime}$ of child health clinics were carried out by clinical medical officers.

An initial pilot survey from several further districts confirmed this variation and it was therefore decided to undertake a fuller study covering as many districts as possible in England.

The original aims of the survey were to try and understand, for each district:

(1) How many preschool medical sessions were carried out relative to the preschool population.

(2) Who, among clinical medical officers, general practitioners, and health visitors, were responsible for doing such sessions.

\section{Method}

Questionnaires were sent to the district medical officers of 178 districts in England, asking:

(1) The 1981 preschool (0-5) population.

(2) The total number of child health clinics for 1981 .

Oxfordshire Health Authority, Radcliffe Infirmary, Oxford OX2 6HE

J A MACFARLANE, MB, MRCP, consultant paediatrician (community child health)

Hillingdon Health Authority, Keeler House, Eastcote, Pinner, Middlesex HA5 1SB

UMSIE PILLAY, $\mathrm{MB}, \mathrm{BCH}$, senior clinical medical officer

Correspondence to: Dr J A Macfarlane.
(3) The number of 1981 child health clinic sessions done by:

(a) clinical medical officers; (b) general practitioners; and $(c)$ health visitors.

Only 178 out of a possible 193 questionnaires were sent because not all the names and addresses of district medical officers in the Mersey and North Western regions were available at the beginning of the survey.

In order to provide a ratio that was comparable between the different districts the total preschool child population was divided by the number of child health clinic sessions held. At the same time, the percentage of child health clinics held by clinical medical officers, general practitioners, and health visitors alone were analysed for each district.

After this information was collected the Department of Health and Social Security was contacted to see what comparable information was available for the same year from its forms LHS $27 / 2$.

\section{Results}

138 districts out of the 178 contacted provided adequate information for analysis. The ratio of the total number of preschool children per child health clinic sessions for all these districts was $9 \cdot 1$, with a standard deviation of 3.3. Fifty three $(38 \cdot 4 \%)$ had figures above this mean. As a result of the enormous variation in the ratio between the districts, rather than presenting all the results we have presented the 10 districts that have the lowest ratio-that is, have the highest number of child health clinics per head of preschool population (table I) compared to the 10 districts which have the highest ratio-that is, have the lowest number of child health clinics per head of preschool population (table II). The comparable ratios worked out from the DHSS's figures (LHS $27 / 2$ ) are also presented. Given that the sets of figures were collected entirely independently, the correlations, with some exceptions, are on the whole good. What is most notable from these figures is that there seems to be a six to sevenfold difference in the density of the preschool child health services between districts.

Information about the number of sessions in each district carried out by clinical medical officers, general practitioners, and health visitors was also collected. This again shows enormous variation so we have given the 10

TABLE I-Districts with the lowest ratio of preschool population per session

\begin{tabular}{|c|c|c|c|c|c|c|}
\hline \multirow[b]{2}{*}{ District } & \multicolumn{2}{|c|}{$\begin{array}{c}\text { Preschool population } \\
(000 \mathrm{~s})\end{array}$} & \multicolumn{2}{|c|}{$\begin{array}{l}\text { Child health clinic } \\
\text { session }\end{array}$} & \multicolumn{2}{|c|}{ Ratio } \\
\hline & Study & LHS $27 / 2$ & Study & LHS $27 / 2$ & Study & LHS $27 / 2$ \\
\hline $\begin{array}{l}\text { Paddington and North Kensington } \\
\text { Brighton } \\
\text { Wirral } \\
\text { Newhaven } \\
\text { Bromley } \\
\text { Frenchay } \\
\text { Salisbury } \\
\text { Tower Hamlets } \\
\text { Isle of Wight } \\
\text { Camberwell }\end{array}$ & $\begin{array}{r}6 \cdot 3 \\
7 \cdot 8 \\
22 \cdot 1 \\
14 \cdot 8 \\
16 \cdot 1 \\
13 \cdot 6 \\
5 \cdot 1 \\
9 \cdot 5 \\
5 \cdot 5 \\
13.6\end{array}$ & $\begin{array}{r}6 \cdot 1 \\
14 \cdot 7 \\
22 \cdot 6 \\
15 \cdot 9 \\
16 \cdot 2 \\
13 \cdot 3 \\
7 \cdot 1 \\
10 \cdot 7 \\
6 \cdot 0 \\
13 \cdot 8\end{array}$ & $\begin{array}{l}1768 \\
1907 \\
4819 \\
3198 \\
3426 \\
2860 \\
1093 \\
1995 \\
1127 \\
2601\end{array}$ & $\begin{array}{l}2248 \\
1959 \\
5817 \\
1754 \\
3555 \\
2860 \\
1056 \\
2255 \\
584 \\
2678\end{array}$ & $\begin{array}{l}3 \cdot 6 \\
4 \cdot 1 \\
4 \cdot 4 \\
4 \cdot 7 \\
4 \cdot 7 \\
4 \cdot 7 \\
4 \cdot 7 \\
4 \cdot 8 \\
4 \cdot 9 \\
5 \cdot 2\end{array}$ & $\begin{array}{r}2 \cdot 7 \\
7 \cdot 5 \\
3 \cdot 9 \\
9 \cdot 1 \\
4 \cdot 6 \\
4 \cdot 6 \\
6 \cdot 7 \\
4 \cdot 7 \\
10 \cdot 3 \\
5 \cdot 1\end{array}$ \\
\hline
\end{tabular}

TABLE II-Districts with the highest ratio of preschool population per session

\begin{tabular}{|c|c|c|c|c|c|c|}
\hline \multirow[b]{2}{*}{ District } & \multicolumn{2}{|c|}{ Preschool } & \multicolumn{2}{|c|}{$\begin{array}{l}\text { Child health clinic } \\
\text { session }\end{array}$} & \multicolumn{2}{|c|}{ Ratio } \\
\hline & Study & LHS $27 / 2$ & Study & LHS $27 / 2$ & Study & LHS $27 / 2$ \\
\hline $\begin{array}{l}\text { West Berkshire } \\
\text { Somerset } \\
\text { Scunthorpe } \\
\text { Barnsley } \\
\text { Huntingdon } \\
\text { Central Birmingham } \\
\text { Portsmouth } \\
\text { Bradford } \\
\text { North west Herts } \\
\text { West Cumbria }\end{array}$ & $\begin{array}{l}27 \cdot 6 \\
20 \cdot 7 \\
12 \cdot 0 \\
14 \cdot 5 \\
11 \cdot 0 \\
14 \cdot 0 \\
30 \cdot 1 \\
27 \cdot 3 \\
8 \cdot 8 \\
8 \cdot 3\end{array}$ & $\begin{aligned} 26 \cdot 1 \\
22 \cdot 0 \\
12 \cdot 5 \\
13 \cdot 9 \\
8 \cdot 6 \\
13 \cdot 4 \\
31 \cdot 0 \\
26 \cdot 6 \\
15 \cdot 9 \\
8 \cdot 7\end{aligned}$ & $\begin{array}{r}1250 \\
1191 \\
689 \\
882 \\
697 \\
889 \\
1924 \\
1789 \\
592 \\
558\end{array}$ & $\begin{array}{r}2195 \\
1027 \\
1485 \\
1759 \\
697 \\
1070 \\
3087 \\
1821 \\
1900 \\
713\end{array}$ & $\begin{array}{l}22 \cdot 0 \\
17 \cdot 4 \\
17 \cdot 4 \\
16 \cdot 4 \\
15 \cdot 8 \\
15 \cdot 6 \\
15 \cdot 0 \\
14 \cdot 9 \\
14 \cdot 9 \\
14 \cdot 7\end{array}$ & $\begin{array}{r}11 \cdot 9 \\
21 \cdot 4 \\
8 \cdot 4 \\
7 \cdot 9 \\
12 \cdot 3 \\
12 \cdot 5 \\
10 \cdot 0 \\
14 \cdot 6 \\
8 \cdot 4 \\
12 \cdot 2\end{array}$ \\
\hline
\end{tabular}

TABLE III-Districts with the highest and lowest participation of clinical medical officers

\begin{tabular}{|c|c|c|c|c|c|}
\hline \multicolumn{3}{|c|}{ Highest } & \multicolumn{3}{|c|}{ Lowest } \\
\hline District & $\%$ study & $\%$ LHS $27 / 2$ & District & $\because$ study & $"$ LHS 27/2 \\
\hline $\begin{array}{l}\text { Victoria } \\
\text { Croydon } \\
\text { Harrow } \\
\text { Hampstead } \\
\text { Enfield } \\
\text { Leeds Eastern } \\
\text { Harrogate } \\
\text { Gateshead } \\
\text { North west Surrey } \\
\text { Haringey } \\
\text { Hillingdon }\end{array}$ & $\begin{array}{l}100 \\
100 \\
96 \cdot 1 \\
86 \cdot 1 \\
96 \cdot 0 \\
92 \cdot 9 \\
92 \cdot 8 \\
92 \cdot 3 \\
91 \cdot 8 \\
90 \cdot 0 \\
89 \cdot 5\end{array}$ & $\begin{array}{l}90 \cdot 6 \\
93 \cdot 6 \\
95 \cdot 7 \\
82 \cdot 9 \\
94 \cdot 0 \\
72 \cdot 9 \\
92 \cdot 8 \\
92 \cdot 7 \\
71 \cdot 9 \\
90 \cdot 9 \\
88 \cdot 1\end{array}$ & $\begin{array}{l}\text { Airedale } \\
\text { East Suffolk } \\
\text { West Suffolk } \\
\text { Somerset } \\
\text { Oxford } \\
\text { Southend } \\
\text { Hull } \\
\text { Sandiwell } \\
\text { Huntingdon } \\
\text { Wakefield }\end{array}$ & \begin{tabular}{r|}
$4 \cdot 8$ \\
8.5 \\
$13 \cdot 7$ \\
$11 \cdot 0$ \\
$16 \cdot 8$ \\
$20 \cdot 2$ \\
$23 \cdot 7$ \\
$28 \cdot 2$ \\
$29 \cdot 7$ \\
$32 \cdot 0$
\end{tabular} & $\begin{array}{r}4 \cdot 8 \\
7 \cdot 2 \\
10 \cdot 9 \\
6 \cdot 2 \\
16 \cdot 8 \\
15 \cdot 9 \\
32 \cdot 2 \\
38 \cdot 3 \\
29 \cdot 7 \\
29 \cdot 0\end{array}$ \\
\hline
\end{tabular}


districts with the highest and lowest participation of clinical medical officers (table III), the highest and lowest participation of general practitioners (table IV), and the highest and lowest participation of health visitors (table $\mathrm{V}$ ).

Table VI shows all the districts in which child health clinics were carried out by medical staff other than clinical medical officers or general practitioners. These were all hospital staff of either senior house officer or registrar grade.

The ratios of child health clinic services relative to preschool population for regions were arrived at by taking an average of the sum of the district figures in that region. These are shown in table VII. Here, interestingly, no distinct pattern emerges and therefore the enormous district variation evens out when looked at on a regional basis. Equivalent figures arrived at from forms LHS 27/2 are shown on the same table. Table VIII shows figures for clinical medical officer, general practitioner, and health visitor participation in the clinics, on a regional basis, along with the DHSS's figures.

\section{Discussion}

Within the National Health Service it might be supposed that there should be some agreement as to what form child health surveillance should take. The recent discussions on the subject, however, have highlighted the fact that this is obviously not so, and is clearly reflected in the district variation demonstrated here.

It is reasonable that some districts might need more child health clinics relative to their population than others, dependent on such factors as degree of urbanisation, percentage

TABLE IV-Districts with the highest and lowest participation of general practitioners

\begin{tabular}{|c|c|c|c|c|c|}
\hline \multicolumn{3}{|c|}{ Highest } & \multicolumn{3}{|c|}{ Lowest } \\
\hline District & ${ }^{\circ}$ o study & $0_{0}^{\circ}$ LHS $27 / 2$ & District & $\%$ study & $\%$ LHS $27 / 2$ \\
\hline $\begin{array}{l}\text { Oxford } \\
\text { Hull } \\
\text { Hastings } \\
\text { Sandiwell } \\
\text { Rotherham } \\
\text { Tower Hamlets } \\
\text { Sunderland } \\
\text { Islington } \\
\text { Bromley }\end{array}$ & $\begin{array}{l}72 \cdot 7 \\
57 \cdot 5 \\
42 \cdot 3 \\
41 \cdot 8 \\
38 \cdot 6 \\
38 \cdot 0 \\
36 \cdot 2 \\
36 \cdot 0 \\
36 \cdot 0\end{array}$ & $\begin{array}{l}72 \cdot 7 \\
48 \cdot 3 \\
27 \cdot 7 \\
39 \cdot 6 \\
35 \cdot 4 \\
31 \cdot 0 \\
32 \cdot 6 \\
29 \cdot 4 \\
29 \cdot 4\end{array}$ & $\begin{array}{l}\text { Essex } \\
\text { East Suffolk } \\
\text { South Cumbria } \\
\text { North Herts } \\
\text { West Essex } \\
\text { Basildon } \\
\text { Frenchay } \\
\text { Kettering } \\
\text { Blackburn } \\
\text { Merton and Sutton }\end{array}$ & $\begin{array}{l}0 \cdot 4 \\
0 \cdot 6 \\
1 \cdot 1 \\
1 \cdot 2 \\
1 \cdot 7 \\
1 \cdot 7 \\
1 \cdot 7 \\
1.6 \\
1.9 \\
2 \cdot 6\end{array}$ & $\begin{array}{l}0.4 \\
0.4 \\
3.5 \\
0.4 \\
1.5 \\
1.5 \\
1.7 \\
1.6 \\
1.8 \\
5 \cdot 0\end{array}$ \\
\hline
\end{tabular}

TABLE V-Districts with the highest and lowest participation of health visitors

\begin{tabular}{|c|c|c|c|c|c|}
\hline \multicolumn{3}{|c|}{ Highest } & \multicolumn{3}{|c|}{ Lowest } \\
\hline District & $\%$ study & ${ }^{\circ}{ }_{0}$ LHS $27 / 2$ & District & ${ }^{\circ}$ study & $\therefore$ LHS $27 / 2$ \\
\hline $\begin{array}{l}\text { East Suffolk } \\
\text { West Suffolk } \\
\text { Airedale } \\
\text { Somerset } \\
\text { Mid-Essex } \\
\text { West Birmingham } \\
\text { Wakefield } \\
\text { Newham } \\
\text { Central Nottingham } \\
\text { Frenchay }\end{array}$ & $\begin{array}{l}90 \cdot 9 \\
86 \cdot 3 \\
90 \cdot 5 \\
72 \cdot 8 \\
68 \cdot 9 \\
64 \cdot 4 \\
64 \cdot 0 \\
62 \cdot 5 \\
60 \cdot 5 \\
60 \cdot 4\end{array}$ & $\begin{array}{l}92 \cdot 4 \\
89 \cdot 1 \\
90 \cdot 5 \\
59 \cdot 3 \\
74 \cdot 8 \\
62 \cdot 5 \\
66 \cdot 9 \\
27 \cdot 8 \\
26 \cdot 9 \\
60 \cdot 4\end{array}$ & $\begin{array}{l}\text { Hillingdon } \\
\text { Grimsby } \\
\text { Tower Hamlets } \\
\text { City and Hackney } \\
\text { Hampstead } \\
\text { Harrow } \\
\text { Islington } \\
\text { Camberwell } \\
\text { North West Herts }\end{array}$ & $\begin{array}{l}1 \cdot 5 \\
1 \cdot 6 \\
2 \cdot 6 \\
3 \cdot 3 \\
3 \cdot 9 \\
3 \cdot 4 \\
4 \cdot 1 \\
4 \cdot 7 \\
4 \cdot 9\end{array}$ & $\begin{array}{l}1 \cdot 9 \\
26 \cdot 1 \\
4 \cdot 6 \\
1 \cdot 7 \\
6 \cdot 5 \\
4 \cdot 3 \\
3 \cdot 4 \\
2 \cdot 8 \\
19 \cdot 7\end{array}$ \\
\hline
\end{tabular}

TABLE VI-Districts in which child health clinics were also done by hospital staff

\begin{tabular}{|c|c|c|c|c|c|}
\hline \multicolumn{3}{|c|}{ Highest } & \multicolumn{3}{|c|}{ Lowest } \\
\hline District & ${ }_{0}^{\circ}$ study & LHS $27 / 2$ & District & ostudy & LHS $27 / 2$ \\
\hline $\begin{array}{l}\text { Hull } \\
\text { South east Staffs } \\
\text { South Lincolnshire } \\
\text { Tower Hamlets }\end{array}$ & $\begin{array}{l}9 \cdot 3 \\
6 \cdot 5 \\
4 \cdot 8 \\
4 \cdot 4\end{array}$ & $\begin{array}{r}10 \cdot 6 \\
6 \cdot 3 \\
3 \cdot 0 \\
3 \cdot 1\end{array}$ & $\begin{array}{l}\text { North Lincoln } \\
\text { York } \\
\text { Rotherham } \\
\text { Dartford }\end{array}$ & $\begin{array}{l}3 \cdot 8 \\
3 \cdot 0 \\
1 \cdot 9 \\
1 \cdot 2\end{array}$ & $\begin{array}{l}3 \cdot 5 \\
3 \cdot 0 \\
5 \cdot 2 \\
1 \cdot 2\end{array}$ \\
\hline
\end{tabular}

Furthermore, the variations between districts are not only as to how much surveillance is carried out but also as to who actually does it. Therefore, it must be asked:

(1) Why is there so much variation? Is a district policy on child health surveillance simply dependent on the whim of those administratively responsible, or is it based on such sound data as is available?

(2) Do we have any evidence that the policy, say, in East Suffolk, which runs relatively few child health clinic sessions per head of preschool population and virtually all done by health visitors, provides any worse child health surveillance than, say, Salisbury which provides three times as many child health clinics per head of preschool population and over $60 \%$ of which are done by doctors?

(3) Given that health visitors are already more concerned than general practitioners in child health surveillance-if there is to be less participation by clinical medical officersshould not their work be undertaken by health visitors rather than general practitioners?

We would therefore suggest that all those concerned-the Royal College of General Practitioners, the British Paediatric Association, the British Medical Association, the Faculty of Community Medicine, and the Health Visitors Association, should meet and jointly agree on a national policy of child health surveillance, based on such evidence as is available at the moment, and that such a policy should be continuously evaluated.

\section{Reference}

'Royal College of General Practitioners. Healthier Children-thinking prevention. Report from general practice 22. London: Royal College of Genera Practitioners, 1982 .

(Accepted 22 May 1984) 